\title{
BMJ Open Poststroke depression and risk of stroke recurrence and mortality: protocol of a meta-analysis and systematic review
}

\author{
Wa Cai, ${ }^{1}$ Robert Stewart, ${ }^{2,3}$ Christoph Mueller, ${ }^{2,3}$ Yi-Jing Li, ${ }^{1}$ Wei-Dong Shen ${ }^{1}$
}

To cite: Cai W, Stewart $R$, Mueller C, et al. Poststroke depression and risk of stroke recurrence and mortality: protocol of a meta-analysis and systematic review. BMJ Open 2018;8:e026316. doi:10.1136/ bmjopen-2018-026316

- Prepublication history for this paper is available online. To view these files, please visit the journal online (http://dx.doi org/10.1136/bmjopen-2018026316).

Received 28 August 2018 Revised 29 0ctober 2018 Accepted 30 October 2018
Check for updates

(c) Author(s) (or their employer(s)) 2018. Re-use permitted under CC BY-NC. No commercial re-use. See rights and permissions. Published by BMJ.

${ }^{1}$ Institute of Acupuncture and Anesthesia, Shanghai Shuguang Hospital Affiliated to Shanghai University of Traditional Chinese Medicine, Shanghai, China ${ }^{2}$ Biomedical Research Centre, South London and Maudsley NHS Foundation Trust, London, UK

${ }^{3}$ Institute of Psychiatry,

Psychology and Neuroscience, King's College London, London, UK

Correspondence to

Dr Wei-Dong Shen;

shenweidong_1018@163.com

\section{ABSTRACT}

Introduction A number of observational studies have indicated that poststroke depression could increase the risk of stroke outcomes. There is a meta-analysis indicating that poststroke depression is a risk factor of all-cause mortality. This paper reports the protocol for a systematic review and meta-analysis to clarify the associations of poststroke depression with stroke recurrence and mortality in order to determine whether poststroke depression is a predictor of stroke outcomes according to data extracted from relevant observational studies.

Methods and analysis MEDLINE, Web of Science databases, EMBASE, Cochrane Central Register of Controlled Trials, Cochrane Database of Systematic Reviews will be used to conduct the search. Published studies written in English will be included. The risk of bias for the studies included in the systematic review or meta-analysis will be assessed by the Newcastle-0ttawa Quality Assessment Scale. HRs for stroke recurrence and mortality with $95 \%$ Cls will be included as primary outcomes. Subgroup analyses and meta-regression will be performed.

Ethics and dissemination Ethics approval will not be needed because the data used in this systematic review will be extracted from published studies. The results of the systematic review focusing on whether depression after stroke is a predictor for stroke recurrence and mortality will be disseminated by publication in a peer-reviewed journal.

PROSPERO registration number CRD42018107944.

\section{INTRODUCTION}

Stroke, also called cerebrovascular accident, is a leading cause of long-term disability and the fourth most common cause of death. Established risk factors for stroke include high plasma cholesterol, high blood pressure, diabetes, atrial fibrillation, race, smoking and older age. ${ }^{1}$ Stroke has been reported to produce increased oxidative stress which is closely associated with the development of psychiatric symptoms. $^{2}$

Poststroke depression (PSD), whose major symptoms are melancholia, dysphoria and vegetative signs such as sleep disorders, reduced libido and energy level, ${ }^{3-5}$ is a

\section{Strengths and limitations of this study}

The meticulous design and use of standardised study rating instruments.

- Compliance with all relevant guidelines for systematic reviews and meta-analyses.

- Different designs and participants' characteristics of all the included studies will lead to high heterogeneity that may limit the quality of the evidence of this meta-analysis and systematic review.

- Limitations will be minimised and strengths enhanced in order to achieve a robust estimation of the association of interest.

significant neuropsychiatric manifestation as it predicts poor outcomes after stroke such as limitations in daily activities, ${ }^{6}$ cognitive disorders, ${ }^{78}$ poor rehabilitation outcomes and social isolation. PSD has a prevalence of approximately $30 \%$ in unselected stroke survivors based on the results of two previous meta-analyses. ${ }^{910}$

Several recent observational studies, including prospective/retrospective cohort studies, have assessed the association between PSD and subsequent risks of stroke morbidity and mortality ${ }^{11-17}$; however, the results of these studies are inconsistent. There is only one previous meta-analysis including 13 studies indicating that depression after stroke is closely associated with increased risk of all-cause mortality. ${ }^{18}$ Notably, there is a lack of a robust meta-analysis focusing on the impact of PSD on stroke recurrence and mortality based on all related studies.

\section{OBJECTIVE}

The protocol study is designed to establish an explicit methodology for conducting a systematic review and meta-analysis, the aims of which comprise:

1. Clarification of the relationship between depression and stroke recurrence as well as mortality in the poststroke patient 


\begin{tabular}{ll}
\hline Table 1 Proposed search terms \\
\hline Theme & Search terms \\
\hline Poststroke depression & 'post stroke depression' \\
& OR 'post stroke depressive \\
& disorder' \\
& OR 'depression after stroke' \\
& OR 'depressive disorder after \\
& stroke' \\
& 'stroke' \\
& OR 'cerebrovascular accident' \\
& OR 'cerebrovascular disease' \\
& OR 'cerebral ischemia' \\
Stroke & OR 'ischemic stroke' \\
& OR 'hemorrhagic stroke' \\
& OR 'Transient Ischemic Attacks' \\
& OR 'mortality' \\
& OR 'all-cause mortality' \\
& OR 'stroke mortality' \\
OR 'cerebrovascular mortality' \\
OR 'death' \\
OR 'stroke death' \\
OR 'cerebrovascular death' \\
'observational study' \\
OR 'prospective cohort study' \\
OR 'retrospective cohort study' \\
OR 'population-based study' \\
\hline Observational study
\end{tabular}

population according to data from observational studies.

2. The analysis of PSD as a predictor of stroke recurrence and mortality.

\section{METHODS AND ANALYSIS}

\section{Review design}

Preferred Reporting Items for Systematic Reviews and Meta-Analyses Protocols (PRISMA-P) ${ }^{19}$ will be set as a guide book for the protocol, and the review methods will be designed based on the Meta-analysis of observational studies in epidemiology: a proposal for reporting, ${ }^{20}$ PRISMA $^{21}$ and Cochrane Collaboration Handbook.

\section{Search strategy}

We will conduct the literature search using Medline (via PubMed), Web of Science databases, EMBASE, Cochrane Central Register of Controlled Trials and the Cochrane Database of Systematic Reviews. Articles published will be searched until the meta-analysis is finished. As shown in table 1, the first theme is PSD, the second theme is stroke and the third theme is observational study. The exploded versions of Medical Subject Headings of each theme will be included. All the three search themes will be combined using the Boolean operator 'and'.

\section{Inclusion criteria}

All observational studies on the association between PSD and stroke recurrence outcomes that meet the following criteria will be included:

1. Prospective or retrospective observational studies.

2. Studies that include stroke outcomes such as ischaemic stroke, haemorrhagic stroke, transient ischemic attacks (TIA), stroke mortality and all-cause mortality.

3. Depression will be ascertained using an appropriate instrument which might include (but will not be limited to) WHO International Classification of Diseases (WHO ICD) or Diagnostic and Statistical Manual of Mental Disorders codes, or research scales such as the Center for Epidemiologic Studies Depression Scale, Patient Health Questionnaire-9, Hospital Anxiety and Depression Scale and Hamilton Depression Rating Scale; alternatively unstructured physician-assigned diagnoses may be used. History of stroke or stroke recurrence may be diagnosed using (but not limited to) WHO ICD codes, National Institutes of Health Stroke Scale (NIHSS) or by physician-assigned codes.

4. Outcomes measured using univariate and multivariate Cox proportional hazards models.

5. Population of adults aged $\geq 18$.

6. Studies published in English.

The PRISMA flow chart shows the process of study selection (figure 1).

\section{Data collection and management}

We will use Endnote (V.x7) to manage the search results and perform screening. The data will be extracted on Microsoft Excel 2007, and statistical analysis will be carried out using STATA V.13 and Review Manager V.5.3 software.

\section{Data extraction}

According to the inclusion criteria, titles and abstracts will be independently checked by two reviewers to identify eligible studies. After that, the full manuscripts of the identified studies will be examined by two researchers who will finally determine the included and excluded studies and clarify the reasons of exclusion. A third researcher will be asked to check, with discussion and consensus sought in cases of disagreement. Two researchers will independently extract the data on author information, publication year, country, follow-up years, number/age/gender of participants, the number of individuals with the outcome based on the exposure (number of deaths in individuals with depression and number of deaths in individuals without depression), the main exposure definition(s) (depression scales or clinician diagnosis), the main outcome definition(s) (stroke recurrence morbidity or mortality, types), the size of the association (HRs with 95\% CI) and factors adjusted for, multiple models if reported, the account taken of important confounders including setting (institutionalised vs community-dwelling), depression measurement time points, stroke severity and activities of daily living. A data extraction sheet will be made to 


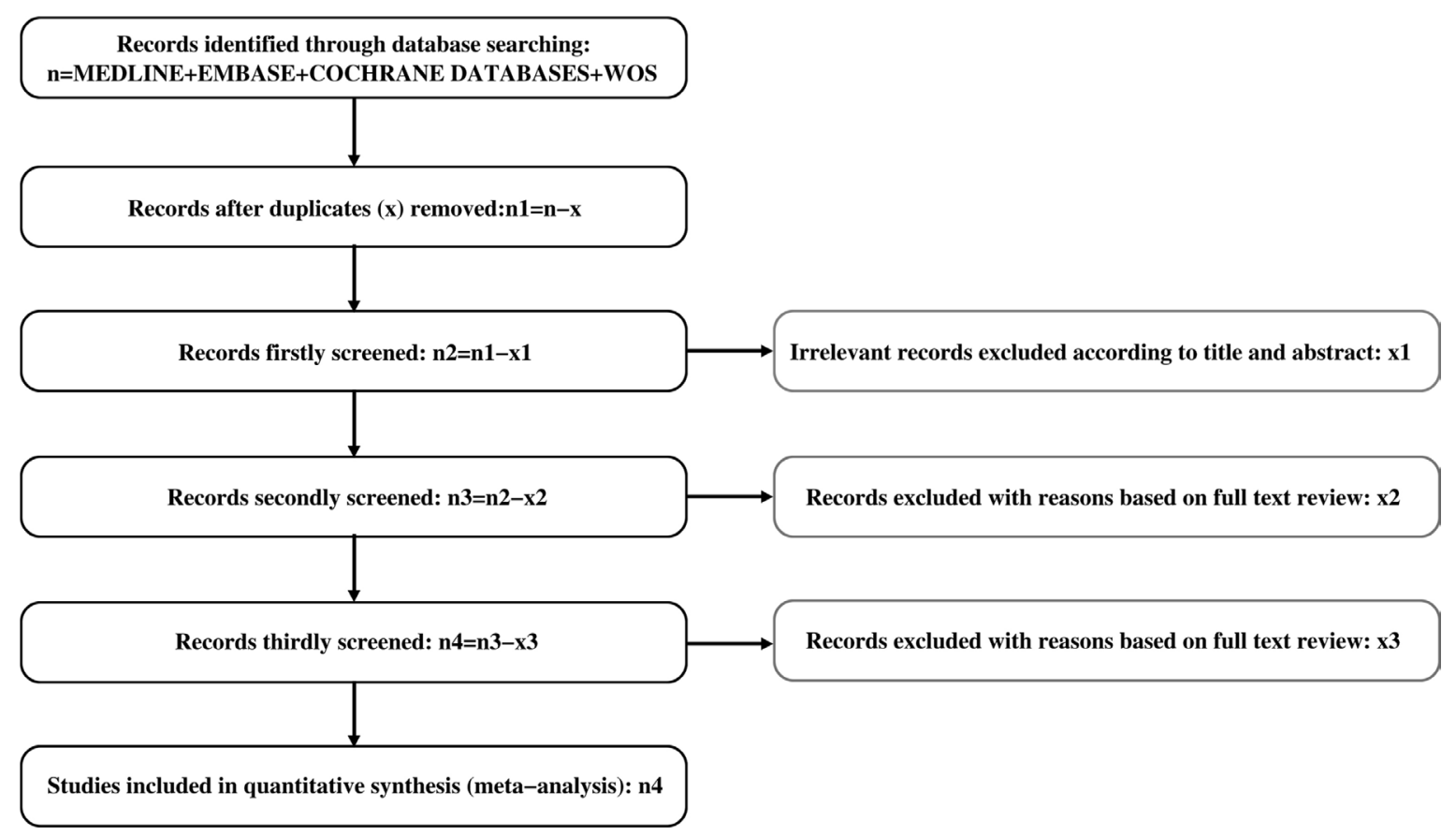

Figure 1 The Preferred Reporting Items for Systematic Reviews and Meta- Analyses flow chart of study selection.

include all the information mentioned above, and it may be adapted during the extraction process in case relevant information is lacking. Authors of the included studies will be contacted in case of missing key information.

\section{Outcomes and prioritisation}

The primary outcome of the meta-analysis is first recorded stroke recurrence or death with PSD diagnosis. Observational studies that calculate effect estimates as HRs are eligible in our meta-analysis. Where included studies report associations between continuous scores and the outcome (such as HR of stroke recurrence per point increase in a depression scale score), those HRs will be included in subgroup analysis according to different severity levels of depression assessed by scale scores. Types of stroke (ischaemic or haemorrhagic) and causes of mortality (stroke-related or all-cause) will be categorised.

\section{Risk of bias assessment in the included studies}

All the included studies will be assessed by the NewcastleOttawa Quality Assessment Scale adapted for cohort studies, the score range of which is $0-9$. Studies with scores $\geq 7$ are conventionally considered as high quality. Two researchers will conduct study quality assessments independently. Any disagreement will be resolved by consultation with a third researcher or by group discussion.

\section{Data synthesis}

A table will be created to summarise characteristics of the included studies. After the data extraction has been completed, researchers will determine the possibility of conducting a meta-analysis. A meta-analysis will be conducted if at least six observational studies using HRs for stroke outcomes and mortality are included.
The extracted HRs with $95 \%$ CIs will be combined by using a random-effects model if it is possible to conduct a meta-analysis. The heterogeneity of the studies will be measured with an $\mathrm{I}^{2}$ value $\left(\mathrm{I}^{2}\right.$ values of $<25 \%, 25 \%-50 \%$ and $>50 \%$ represent low, medium and high heterogeneity, respectively ${ }^{22}$ ). Adjusted and unadjusted estimates will be compared separately for each outcome. The pooled HRs and 95\% CIs will be the primary outcome of the meta-analysis. A narrative synthesis will be taken if a meta-analysis is not possible. Visual assessment of the funnel plot and Egger's test will be used to assess publication bias. ${ }^{23}$ The Grading of Recommendations Assessment, Development and Evaluation tool will be used to evaluate the strength of the evidence body. ${ }^{24}$

In order to clarify major factors underlying any high heterogeneity, subgroup analyses and meta-regression will be performed according to the duration of follow-up (above/below median years), stroke severity (low, moderate, severe according to NIHSS), definition of depression (WHO ICD codes, scales, physician diagnosis), study setting (institutionalised vs community-dwelling), bias score (above/below median scores), the stroke recurrence outcome type (ischaemic stroke, haemorrhagic stroke), mortality type (stroke-specified, all-cause), mean age (above/below median years old) / male proportion of study participants (above/below median \%) and sample size (above/below median numbers). Sensitivity analyses will be conducted by excluding studies one by one and comparing the results in the analysis.

\section{Patient and public involvement}

No patients will be involved in this study. 


\section{DISCUSSION}

PSD as a predictor of stroke recurrence and mortality remains to be confirmed by an updated meta-analysis because the last one, to our knowledge, was carried out 5 years ago. ${ }^{18}$ Thus, a meta-analysis and systematic review will be designed by us to determine whether the risk of stroke recurrence and mortality outcomes is relatively high in people with PSD.

Strengths and limitations will be highlighted in the process of identified evidence. The meticulous design, the use of standardised study rating instruments and compliance with all relevant guidelines for systematic reviews and meta-analyses are anticipated strengths. Limitations will mainly originate from different designs and characteristics of all the included studies; this may lead to high heterogeneity which will in turn lower the quality of the evidence of this meta-analysis and systematic review; however, this may be overcome by including subgroup analyses and meta-regression in the meta-analysis.

Significantly, the association between PSD and stroke recurrence and mortality will be clarified in the results of the designed meta-analysis and systematic review, seeking to minimise its limitations and enhance its strengths, as mentioned.

\section{Ethics and dissemination}

There will be no concerns about privacy. The systematic review will focus on whether PSD is a predictor for stroke recurrence and mortality outcomes, the results of which will be disseminated by publication in a peer-reviewed journal after completion.

Acknowledgements The authors would like to thank China Scholarship Council for support of the collaborations between Shanghai University of Traditional Chinese Medicine and King's College London.

Contributors WC and W-DS designed the study. WC was the principal investigator and guarantor. WC, Y-JL and W-DS conducted the study. RS and CM gave statistical and epidemiological support. WC drafted the article with the support of W-DS.

Funding WC are funded by Graduate innovation training program, Shanghai University of traditional Chinese Medicine (Grant No. Y201805) . W-DS and Y-JL are funded by Shanghai Committee of Science and Technology, China (Grant No. 16401970402/18401970601), Three-year action plan for the development of Traditional Chinese medicine, Shanghai, China (Grant No. ZYSNXD-CCHPGC-JD-014) and Shanghai Municipal Commission of Health and Family Planning, China (Grant No. ZYKC201701001). RS and CM are part-funded by the National Institute for Health Research (NIHR) Biomedical Research Centre at South London and Maudsley NHS Foundation Trust and King's College London.

Competing interests None declared.

Patient consent for publication Not required.

Ethics approval Ethics approval will not be needed because the data used in this systematic review will be extracted from published studies.

Provenance and peer review Not commissioned; externally peer reviewed.

Open access This is an open access article distributed in accordance with the Creative Commons Attribution Non Commercial (CC BY-NC 4.0) license, which permits others to distribute, remix, adapt, build upon this work non-commercially, and license their derivative works on different terms, provided the original work is properly cited, appropriate credit is given, any changes made indicated, and the use is non-commercial. See: http://creativecommons.org/licenses/by-nc/4.0/.

\section{REFERENCES}

1. Goldstein LB, Bushnell CD, Adams RJ, et al. Guidelines for the primary prevention of stroke: a guideline for healthcare professionals from the American Heart Association/American Stroke Association. Stroke 2011;42:517-84.

2. Bolaños JP, Moro MA, Lizasoain I, et al. Mitochondria and reactive oxygen and nitrogen species in neurological disorders and stroke: therapeutic implications. Adv Drug Deliv Rev 2009;61:1299-315.

3. Whyte EM, Mulsant BH. Post stroke depression: epidemiology, pathophysiology, and biological treatment. Biol Psychiatry 2002;52:253-64.

4. Tateno A, Kimura M, Robinson RG. Phenomenological characteristics of poststroke depression: early- versus late-onset. Am J Geriatr Psychiatry. Sep 2002;10:575-82.

5. Paradiso S, Vaidya J, Tranel D, et al. Nondysphoric depression following stroke. J Neuropsychiatry Clin Neurosci 2008;20:52-61.

6. Pohjasvaara T, Vataja R, Leppävuori A, et al. Depression is an independent predictor of poor long-term functional outcome poststroke. Eur J Neurol 2001;8:315-9.

7. Chemerinski E, Robinson RG, Kosier JT. Improved recovery in activities of daily living associated with remission of poststroke depression. Stroke 2001;32:113-7.

8. Serrano S, Domingo J, Rodríguez-Garcia E, et al. Frequency of cognitive impairment without dementia in patients with stroke: a twoyear follow-up study. Stroke 2007;38:105-10.

9. Hackett ML, Anderson CS. Predictors of depression after stroke: a systematic review of observational studies. Stroke 2005;36:2296-301.

10. Ayerbe L, Ayis S, Wolfe CD, et al. Natural history, predictors and outcomes of depression after stroke: systematic review and metaanalysis. Br J Psychiatry 2013;202:14-21.

11. Liang C, Li M, Zhang X, et al. Association between post-stroke depression in lacunar stroke patients and rehospitalization. Int J Clin Exp Med 2018;11:4126-31.

12. Yu S, Arima H, Bertmar C, et al. Depression but not anxiety predicts recurrent cerebrovascular events. Acta Neurol Scand 2016;134:29-34.

13. Hong JP, Park S, Ahn SH, et al. Factors associated with post-stroke suicidal death. J Psychiatr Res 2018;96:135-7.

14. Freak-Poli R, Ikram MA, Franco OH, et al. Depressive symptoms prior to and after incident cardiovascular disease and long-term survival. A population-based study of older persons. Depress Anxiety 2018;35:18-31.

15. Razmara A, Valle N, Markovic D, et al. Depression is associated with a higher risk of death among stroke survivors. J Stroke Cerebrovasc Dis 2017;26:2870-9.

16. Jørgensen TSH, Wium-Andersen IK, Wium-Andersen MK, et al. Incidence of depression after stroke, and associated risk factors and mortality outcomes, in a large cohort of Danish patients. JAMA Psychiatry 2016;73:1032-40.

17. de Mello RF, Santos IS, Alencar AP, et al. Major depression as a predictor of poor long-term survival in a Brazilian Stroke Cohor (Study of Stroke Mortality and Morbidity in Adults) EMMA study. J Stroke Cerebrovasc Dis 2016;25:618-25.

18. Bartoli F, Lillia N, Lax A, et al. Depression after stroke and risk of mortality: a systematic review and meta-analysis. Stroke Res Treat 2013;2013:1-11.

19. Moher D, Shamseer L, Clarke M, et al. Preferred reporting items for systematic review and meta-analysis protocols (PRISMA-P) 2015 statement. Syst Rev 2015;4:1.

20. Stroup DF, Berlin JA, Morton SC, et al. Meta-analysis of observational studies in epidemiology: a proposal for reporting. Meta-analysis Of Observational Studies in Epidemiology (MOOSE) group. JAMA 2000;283:2008-12.

21. Yoshitoku Y, Toyonori O. Practice guideline of evidence-based medicine: Preferred Reporting Items for Systematic Reviews and Meta-analyses (the PRISMA statement). Inf Process Manag 2011;54:254-66.

22. Melsen WG, Bootsma MC, Rovers MM, et al. The effects of clinical and statistical heterogeneity on the predictive values of results from meta-analyses. Clin Microbiol Infect 2014;20:123-9.

23. Sterne JA, Egger M, Smith GD. Systematic reviews in health care: Investigating and dealing with publication and other biases in metaanalysis. BMJ 2001;323:101-5.

24. Owens DK, Lohr KN, Atkins D, et al. Grading the strength of a body of evidence when comparing medical interventions. Rockville, MD: AHRQ Methods for Effective Health Care, 2008. 